\title{
UNIVERSAL BOUNDS AND MONOTONICITY OF ORDINAL INVARIANTS
}

\author{
JAMES R. BOONE
}

\begin{abstract}
The tightness of a topological space provides a universal bound for all ordinal invariants determined by weak topologies generated by collections of subsets. All ordinal invariants are monotonic decreasing for pseudo-open mappings.
\end{abstract}

I. Introduction. The bounds for the ordinal invariants sequential order $\sigma$ of sequential spaces and compact order $\kappa$ of $k$-spaces have been determined as $\sigma(X) \leqslant \omega_{1}[2]$ and $\kappa(X) \leqslant t(X)^{+}[7]$ where $t(X)^{+}$is the successor of the tightness of $X$. These ordinal invariants are monotonic decreasing for pseudo-open mappings [3] in the sense that, if $X$ is a $k$-space (sequential space) and $f: X \rightarrow Y$ is a continuous pseudo-open surjection then $\kappa(X) \geqslant \kappa(Y)(\sigma(X) \geqslant \sigma(Y))$. Recently in the studies of other ordinal invariants, I determined that these properties of ordinal invariants are valid in the most general setting.

It is the purpose of this paper to present the following very inclusive results. The cardinal successor of the tightness of a topological space, $t(X)^{+}$, provides the universal bound for all ordinal invariants determined by any weak topology on $X$ generated by a collection of subsets. Also, all ordinal invariants are monontonic decreasing for pseudo-open mappings.

II. Preliminaries. The concept of a natural cover introduced by Stan Franklin [8] will be used extensively in this paper, because the results concern any ordinal invariant determined by the weak topology generated by a collection of subsets. The following notions are from [8] where the essential foundations of spaces with the weak topology were presented. A natural cover $\Sigma$ in a space $X$ is a function which assigns each space $X$ to a cover $\Sigma_{X}$ satisfying (a) if $S \in \Sigma_{X}$ and $S$ is homeomorphic to a subspace $T \subset Y$, then $T \in \Sigma_{Y}$ and (b)* if $f: X \rightarrow Y$ is continuous and $S \in \Sigma_{X}$, then $f(S) \in \Sigma_{Y}$. (Condition (b)* is slightly stronger than the condition (b) in [8], which does not require $f(S) \in \Sigma_{Y}$ but only that $f(S) \subset T$ for some $T \in \Sigma_{Y}$. Condition (b)* was needed in the proof of the characterization Lemma 3.2.) Many times the collection of subsets $\Sigma_{X}$ is referred to as sequences, $\Omega$-nets, linearly ordered nets or sets of cardinality $\leqslant m$. A space $X$ has the weak

Received by the editors October 9, 1980 and, in revised form, March 17, 1981.

1980 Mathematics Subject Classification. Primary 54B15; Secondary 54A10, 54A25, 54C10, 54050, 54054.

Key words and phrases. Natural cover, ordinal invariant, tightness, pseudo-open. 
topology generated by the natural cover $\Sigma$ provided $H \subset X$ is closed if and only if $H \cap S$ is closed in $S$ for each $S \in \Sigma$. A set $H$ is $\Sigma$-closed ( $\Sigma$-open) provided $S \cap H$ is closed (open) in $S$ for each $S \in \Sigma$. Thus, if every $\Sigma$-open subset of $X$ is open, then $X$ has the weak topology generated by $\Sigma$ and in this case $X$ is called a $\Sigma$-space [8]. ${ }^{1}$ For example, the $k$-spaces, sequential spaces, $\Omega$-net spaces [10], lo-net spaces [4] and spaces of tightness $m$ [5] are $\Sigma$-spaces. For $A \subset X$, let $\Sigma \operatorname{cl}(A)=$ $\left\{p \in X: p \in \operatorname{cl}_{S}(S \cap A)\right.$ for some $\left.S \in \Sigma\right\}$. Let $A^{0}=A$ and let $A^{1}=\Sigma \operatorname{cl}(A)$. Suppose $A^{\alpha}$ is defined for each ordinal $\alpha<\beta$. If $\beta=\gamma+1$, let $A^{\beta}=\Sigma \operatorname{cl}\left(A^{\gamma}\right)$ and if $\beta$ is a limit ordinal let $A^{\beta}=\cup\left\{A^{\alpha}: \alpha<\beta\right\}$. The $\Sigma$-characteristic of $X$ is $\Sigma(X)=\inf \left\{\beta: A^{\beta}=\operatorname{cl}(A)\right.$, for $\left.A \subset X\right\}$. This $\Sigma$-characteristic of $X$ is the ordinal invariant determined by the weak topology generated by the natural cover $\Sigma$.

III. Theorems. In the following theorem the successor of the tightness of a space $X, t(X)^{+}$, is shown to be the bound for all ordinal invariants defined on $X$ by a weak topology generated by any natural cover. The tightness of $X, t(X)$, [9], is the least infinite cardinal $m$ such that for each $A \subset X$ and for each $p \in \operatorname{cl}(A)$ there is some $B \subset A$ such that $\operatorname{card}(B) \leqslant m$ and $p \in \operatorname{cl}(B)$. It has been shown in [5] that, for a space $X, t(X) \leqslant m$ if and only if $X$ has the weak topology generated by the collection of su sets of cardinality $\leqslant m$ and that tightness is monotonic decreasing for quotient mappings. Franklin [8] has shown that $X$ is a $\Sigma$-space if and only if $\Sigma(X)$ exists.

THEOREM 3.1. If $X$ is a $\Sigma$-space, then $\Sigma(X) \leqslant t(X)^{+}$.

Proof. Suppose $\Sigma(X)>t(X)^{+}$. Let $\mu=t(x)^{+}$. Then there exists $A \subset X$ such that $A^{\mu+1}-A^{\mu} \neq \varnothing$. Let $p \in A^{\mu+1}-A^{\mu}$. Since $A^{\mu+1}=\{r \in X$ : there exists an $S \in \Sigma_{X}$ such that $\left.r \in \operatorname{cl}_{S}\left(S \cap A^{\mu}\right)\right\}$, there exists an $S \in \Sigma_{X}$ such that $p \in$ $\operatorname{cl}_{S}\left(S \cap A^{\mu}\right)-\left(S \cap A^{\mu}\right)$. Since $p \in \operatorname{cl}\left(S \cap A^{\mu}\right)$, there is a set $T \subset S \cap A^{\mu}$ such that $\operatorname{card}(T) \leqslant t(X)<\mu=t(X)^{+}$and $p \in \operatorname{cl}(T)$. For each $q \in T$, there is some $\nu<\mu$ such that $q \in A^{\nu}$. Since $\mu=t(X)^{+}$is a regular cardinal, there exists $\gamma<\mu$ such that $T \subset A^{\gamma}$. Thus $p \in \operatorname{cl}_{S}(T) \subset \operatorname{cl}_{S}\left(S \cap A^{\gamma}\right) \subset A^{\gamma+1}$. Thus, $p \in S \cap A^{\mu}$, which is a contradiction and this completes the proof.

Arhangelskii [1] introduced the concept of a pseudo-open mapping which may be characterized as $f: X \rightarrow Y$ is a pseudo-open mapping if $p \in \operatorname{cl}_{Y}(A)$ implies $f^{-1}(p) \cap \operatorname{cl}_{X}\left(f^{-1}(A)\right) \neq \varnothing$. Modifications of this condition have been used to characterize open mappings and closed mappings [6]. Pseudo-open mappings are simultaneous generalizations of open mappings and closed mappings and the continuous pseudo-open surjections are quotient mappings. In [3] it was shown that the ordinal invariants, sequential order and compact order are monotonic decreasing for pseudo-open mappings. The following lemma is needed to prove the monotonicity theorem. A function $f: X \rightarrow Y$ defined on a space $X$ is $\Sigma$-continuous

${ }^{1}$ These spaces are not the $\Sigma$-spaces of K. Nagami, Fund. Math. 65 (1969), 169-192. 
provided $\left.f\right|_{S}: S \rightarrow Y$ is continuous for each $S \in \Sigma_{X}$. The following characterizations of $\Sigma$-continuity are generalizations of Proposition 3.2, Theorem 3.4 and Corollary 3.5 of [3]. The proof is omitted because it follows directly from the proofs in [3].

LEMMA 3.2. Let $f: X \rightarrow Y$. The following are equivalent:

(a) $f$ is $\Sigma$-continuous.

(b) $f^{-1}(H)$ is $\Sigma$-closed in $X$, for each $\Sigma$-closed set $H \subset Y$.

(c) $f^{-1}(G)$ is $\Sigma$-open in $X$, for each $\Sigma$-open set $G \subset Y$.

(d) $f(\Sigma \operatorname{cl}(A)) \subset \Sigma \operatorname{cl}(f(A))$, for each $A \subset X$.

(e) $\Sigma \operatorname{cl}\left(f^{-1}(B)\right) \subset f^{-1}(\Sigma \operatorname{cl}(B))$, for each $B \subset Y$.

(f) $f\left(A^{\beta}\right) \subset(f(A))^{\beta}$, for each ordinal $\beta$ and $A \subset X$.

(g) $\left(f^{-1}(B)\right)^{\alpha} \subset f^{-1}\left(B^{\alpha}\right)$, for each ordinal $\alpha$ and $B \subset Y$.

Lemma 3.3 [8, Proposition 2.13]. $X$ is a $\Sigma$-space if and only if every $\Sigma$ continuous function defined on $X$ is continuous.

The following theorem extends the monotonicity theorem to all ordinal invariants defined by the weak topology generated by any natural cover.

THEOREM 3.4. If $X$ is a $\Sigma$-space and $f: X \rightarrow Y$ is a continuous pseudo-open surjection, then $Y$ is a $\Sigma$-space and $\Sigma(X) \geqslant \Sigma(Y)$.

Proof. Since the quotient space of a $\Sigma$-space is a $\Sigma$-space [8], $Y$ is a $\Sigma$-space and $\Sigma(Y)$ exists. Suppose $\Sigma(X)=\mu$. Let $B$ be any subset of $Y$. We will show $B^{\mu}$ is closed in $Y$ and it will follow that $\Sigma(Y) \leqslant \mu$. Let $p \in \operatorname{cl}_{X}\left(f^{-1}\left(B^{\mu}\right)\right)$. Then $f(p) \in$ $f\left(\operatorname{cl}_{X}\left(f^{-1}\left(B^{\mu}\right)\right)\right) \subset \operatorname{cl}_{Y}\left(B^{\mu}\right)=\operatorname{cl}_{Y}(B)$. Since $f$ is pseudo-open and $f(p) \in \operatorname{cl}_{Y}(B)$, $f^{-1}(p) \cap \operatorname{cl}_{X}\left(f^{-1}(B)\right) \neq \varnothing$. Since $\Sigma(X)=\mu, \operatorname{cl}_{X}\left(f^{-1}(B)\right)=\left(f^{-1}(B)\right)^{\mu}$ and thus $f^{-1}(f(p)) \cap\left(f^{-1}(B)\right)^{\mu} \neq \varnothing$. Since $f$ is continuous, by Lemma 3.2(g) $\left(f^{-1}(B)\right)^{\mu} \subset$ $f^{-1}\left(B^{\mu}\right)$. Hence $f^{-1}(f(p)) \cap f^{-1}\left(B^{\mu}\right) \neq \varnothing$. Thus $f(p) \cap B^{\mu} \neq \varnothing$ and $p \in f^{-1}\left(B^{\mu}\right)$. Thus, $\operatorname{cl}_{X}\left(f^{-1}\left(B^{\mu}\right)\right) \subset f^{-1}\left(B^{\mu}\right)$ and $f^{-1}\left(B^{\mu}\right)$ is closed. Since $f$ is a continuous, pseudo-open surjection, $f$ is a quotient mapping. Hence $f^{-1}\left(B^{\mu}\right)$ is closed implies $B^{\mu}$ is closed. Thus, for each subset $B \subset Y, B^{\mu}$ is closed. Accordingly, $\Sigma(Y)<\mu$. This completes the proof.

The various theorems concerning the cardinal invariants in sequential and $k$-spaces in [7] are also true in the general setting of natural covers where we would consider the $\Sigma$-cardinal of $X$ and the pointwise $\Sigma$-cardinal of $X$.

\section{REFERENCES}

1. A. Arhangelskii, Some types of factor mappings and the relations between classes of topological spaces, Soviet Math. Dokl. 4 (1963), 1726-1729.

2. A. Arhangelskii and S. P. Franklin, Ordinal invariants for topological spaces, Michigan Math. J. 15 (1968), 313-320.

3. J. R. Boone, Pseudo-open functions are monotonic decreasing for ordinal and cardinal invariants, General Topology Appl. 9 (1978), 243-251.

4. __ A note on linearly ordered net spaces, Pacific J. Math. (to appear).

5. —_ On the tightness of a topological space (submitted). 
6. __ On open mappings, closed mappings and ordinal invariants, Bull. Austral. Math. Soc. 19 (1978), 235-238.

7. J. R. Boone, S. W. Davis and G. Greunhage, Cardinal functions for k-spaces, Proc. Amer. Math. Soc. 68 (1978), 355-358.

8. S. P. Franklin, Natural covers, Compositio Math. 21 (1969), 253-261.

9. I. Juhasz, Cardinal functions in topology, Mathematical Centre Tracts, no. 34, Mathematisch Centrum, Amsterdam, 1971.

10. J. E. Vaughan, Convergence, closed projections and compactness, Proc. Amer. Math. Soc. 51 (1975), 469-476.

Department of Mathematics, Texas AaM University, College Station, Texas 77843 